\title{
Realidade Virtual para saúde no Brasil: conceitos, desafios e oportunidades
}

\author{
Fátima de Lourdes dos Santos Nunes*, Rosa Maria Esteves Moreira da Costa, \\ Liliane dos Santos Machado, Ronei Marcos de Moraes
}

Resumo A Realidade Virtual é uma área tipicamente multidisciplinar que envolve conceitos provenientes das Engenharias, Computação, Física, Artes e também da área específica da aplicação à qual ela se destina. Por permitir uma interação humano-computador mais natural em ambiente tridimensional (3D) e possibilitar a reprodução de situações reais, torna-se um recurso de amplo potencial para a área da saúde. Este artigo aborda conceitos, desafios e oportunidades de aplicações de Realidade Virtual na área de saúde no Brasil, apresentando um estudo sobre as pesquisas na área e aplicações desenvolvidas. A análise da situação atual mostra que há vários grupos brasileiros de pesquisa desenvolvendo aplicações com grande diversidade de assuntos, contemplando principalmente as áreas de treinamento e educação. A partir da análise conduzida, verifica-se que ainda há vários desafios a serem superados a fim de que as aplicações de Realidade Virtual sejam incluídas na rotina dos profissionais da área de saúde. Se por um lado tais desafios retardam o uso efetivo desta tecnologia na prática, por outro constituem oportunidades ímpares de pesquisa e desenvolvimento, conforme é destacado na conclusão deste artigo.

Palavras-chave Realidade Virtual, Educação, Treinamento médico, Desafios, Oportunidades.

\section{Virtual Reality for health care in Brazil: concepts, challenges and opportunities}

\begin{abstract}
Virtual Reality (VR) is a typically multidisciplinary field which involves concepts from Engineering, Computer Science, Physics, Arts as well as concepts from the specific application field. Because VR allows a more natural human-computer interaction in three-dimensional environments and enables the reproduction of real situations, it becomes a resource of wide potential for healthcare field. This paper discusses concepts, challenges and opportunities of Virtual Reality applications for healthcare in Brazil, by presenting a study about researches in the area and developed applications. The analysis of the current situation shows that there are various Brazilian research groups developing applications considering great diversity of subjects, covering mainly training and education area. This analysis shows that there are still many challenges to be overcome so that applications using Virtual Reality can be included in the routine of healthcare professionals. On one hand these challenges hinder the effective use of technology in practice, on the other they open new opportunities for research and development, as is highlighted in the conclusion of this paper.
\end{abstract}

Keywords Virtual Reality, Education, Medical training, Challenges, Opportunities. 


\section{Extended Abstract}

\section{Introduction}

The use of Virtual Reality (VR) applications in different fields of activity has experienced an increase in growth over the last years. Virtual Reality is a multidisciplinary research field, which involves concepts from Engineering, Computer Science, Physics, and Arts as well as concepts from the specific application field. In general, the VR applications explore advanced technologies in relation to interfaces, providing strategies to immerse the user in environments that can be actively interacted with and explored. Therefore, it allows a more natural human-computer interaction in three-dimensional (3D) environments and enables the reproduction of real situations in safe digital environments. These features make VR a resource of wide potential for healthcare field. The required characteristics of $V R$ (immersion, interaction and presence) present special connotations when applications are intended for healthcare. The $3 D$ simulation procedures in health care area have been object of some researches around the world, but until now, we do not have a panorama of the Brazilian results in this context. This paper discusses concepts, challenges and opportunities of Virtual Reality applications for healthcare in Brazil by presenting a study about researches in the area and developed applications.

\section{Material and Methods}

$V R$ applications for healthcare were categorized according to their purpose. Seven categories are briefly described to highlight potentialities in the healthcare area. Thus, researches from Brazilian groups are presented to exemplify applications for education, training, rehabilitation, development tools, programming languages, gaming and collaborative activities. In order to discuss the researches, initiatives and developments in Brazil, we conducted an analysis about the situation of publications in some scientific conferences in the last years. Some important aspects were observed like the number of papers that integrated VR with a health subject and the devices they used. We classified them according to their purposes (education, training, development tools, etc) and we checked if the works had some evaluation process. The analysis focused on conferences related to informatics and health, because there is a restricted presence of consolidated journals with scope in this area in Brazil. Thus, only one journal was considered. We selected the editions from 2004 to 2009 of the "Congresso Brasileiro de Engenharia Biomédica-CBEB" (Brazilian Congress of Biomedical Engineering); the editions from 2004 to 2010 of the "Workshop de Informática Médica-WIM" (Workshop on Medical Informatics); the editions from 2006 to 2010 of the Symposium on Virtual and Augmented Reality-SVR and the editions from 2004 to 2010 of the "Congresso Brasileiro de
Informática em Saúde-CBIS" (Brazilian Congress on Informatics in Heath). Editions from 1999 to 2010 of the Brazilian Journal on Biomedical Engineering were also analyzed.

\section{Results and Discussion}

The analysis of these Brazilian publications indicates that the recent researches in this domain were mainly published in conference proceedings. The only journal analyzed did not present papers in the VR-Health area in the 2004-2010 years. The data indicate that most applications that explores the VR technologies for healthcare use conventional devices and are directed for education and training (53\%), besides they did not present evaluation processes. The main group of researchers involved in these applications is composed by students and professionals from the VR area. However, it was observed an effort related to the development of tools to support the creation of VR applications for training and simulation that could be easily used by professionals from the health area. A critical point is the lack of evaluation of the applications that may influence in the spread and use of them. This lack was observed in $55 \%$ of the analyzed applications, considering the quality requirements like usability, adequacy to functional requirements and effectiveness. Additionally, most of the researches were developed in the academic context without participation of the productive sector. This can explain the use of free or open source software in works related to tools for development of $3 D$ environments. The government is the main sponsor of the researches and this factor stresses the distance of companies in relation to the researches related to VR in healthcare in Brazil. Some challenges for VR in healthcare can be identified from this analysis, like the difficulty of controlling simultaneously several devices, the quality of graphic objects, the need of defining methodologies to evaluate users as well as the performance and the adequacy of applications, the necessity of developing more suitable tangible devices for medical training, ethical considerations involved in the systems development and the multidisciplinary requirements of the researches.

\section{Conclusions}

Taking into account the brazilian researches looking for applications for healthcare using VR and the challenges related to their development, some opportunities for research and development were outlined: the availability of frameworks in order to allow a faster building of applications with low cost; the availability of graphic objects libraries specific for this category of application; the development of hardware for intuitive, ergonomic and precise interaction; simulations of the objects behavior similar to real one and the definition of methodologies for modeling and evaluation in order to support the construction of high quality products. 


\section{Introdução}

Muitas discussões têm sido conduzidas no meio científico com o intuito de definir os desafios sociais, ambientais e econômicos a serem superados e como as diferentes áreas das ciências podem contribuir e cooperar para fomentar o desenvolvimento de soluções inovadoras. Tais discussões despertam a reflexão sobre questões fundamentais relacionadas ao uso das novas tecnologias e como estas podem colaborar na busca de soluções a curto, médio e longo prazo para as diversas áreas dos setores produtivos e econômicos. Neste sentido, as áreas de Engenharia e Computação, considerando especificamente a sociedade brasileira, apresentam desafios como o gerenciamento de grandes volumes de dados, inclusive multimídia e distribuídos, a modelagem de sistemas complexos, a acessibilidade à informação, e a construção de sistemas disponíveis, corretos, seguros, escaláveis, persistentes e ubíquos (Carvalho et al., 2006).

O desenvolvimento tecnológico vem alterando diversas práticas na área de saúde, abrangendo atividades como diagnóstico, terapia, gerenciamento e educação, e exigindo o desenvolvimento de novas habilidades pelos profissionais da área. Mais recentemente, uma nova tecnologia vem permeando a área da saúde, trazendo novas possibilidades de composição, visualização tridimensional de imagens, interação e simulação: a Realidade Virtual, que oferece interfaces avançadas capazes de proporcionar imersão do usuário em ambientes com os quais pode interagir e explorar.

Ao observar a produção científica e comercial de Realidade Virtual (RV) nos últimos anos, é inegável que a área de saúde tem sido um importante campo de aplicação, contribuindo para que a própria tecnologia evolua em termos de hardware e software, de modo a suprir as demandas específicas da área de aplicação. A viabilidade de reproduzir situações reais sem risco a pacientes, a diminuição de custos devido à redução de uso de objetos físicos e a possibilidade de simular e visualizar ações impossíveis de serem percebidas no mundo real constituem motivos para que a RV aplicada à saúde constitua uma área de interesse crescente, com benefícios tanto para a saúde, quanto para o desenvolvimento das áreas tecnológicas.

O objetivo deste artigo é apresentar um panorama das aplicações de RV para a área de saúde e resultados da experiência de grupos de pesquisa do Brasil. Analisando tais resultados, é apresentada uma reflexão sobre a necessidade de interdisciplinaridade, os desafios encontrados para desenvolver ferramentas e as tendências atuais, identificando potencialidades e oportunidades de pesquisa e desenvolvimento.
Na literatura vários trabalhos apresentam estudos sobre o estado da arte em uma determinada área, considerando a situação global ou situações de países específicos (DesRoches et al., 2008; Garg, 2003). Estes trabalhos são importantes para que se tenha idéia dos índices de produção em áreas específicas e para que se possa compará-los com aqueles de outros países.

Especificamente relacionados à área de RV aplicada à saúde, há mais de uma década Chestnut e Crumpton (1997) e Brown et al. (1997) já apresentavam a Realidade Virtual como uma ferramenta com alto potencial de uso para treinamento de procedimentos médicos e para tratamento de diversas deficiências físicas e cognitivas. Mais recentemente, Chodos et al. (2010) apresentaram uma análise de diversas aplicações de simulação usando mundos virtuais para a educação médica. Já o trabalho de Kuljis et al. (2007) discutiu as possibilidades da área de saúde ser beneficiada por métodos de simulação e modelagem, da mesma forma que a área de manufatura tem se beneficiado. Uma das áreas nas quais a RV tem tido uma alta inserção, com resultados bem significativos, é a de reabilitação cognitiva e física de pessoas que sofreram algum tipo de lesão cerebral ou trauma psicológico (D'Angelo et al., 2010; Gerardi et al., 2008; Powell et al., 2010; Rahman et al., 2011). Neste contexto, as novas tecnologias de interação humano-computador, tal como o console de jogos Wii, vêm ampliando ainda mais as perspectivas de uso dos ambientes virtuais por pessoas que sofreram diferentes tipos de traumas (Butler e Willet, 2010). Todas essas áreas podem ser contempladas em jogos com propósitos específicos, que servem tanto para o aprendizado de novos conceitos, quanto para o treinamento ou desenvolvimento de habilidades (Machado et al., 2011).

No caso do presente artigo, o levantamento dos trabalhos que exploram a tecnologia de RV na área da saúde possibilita tecer um panorama dos trabalhos realizados no Brasil, ressaltando as dificuldades e as necessidades, que por sua vez, podem se constituir em oportunidades de pesquisa e desenvolvimento.

\section{Características da Realidade Virtual aplicada à saúde}

Aplicações baseadas em RV permitem aos usuários executar navegação e interação em tempo real, em ambientes 3D gerados por computador, usando canais multissensoriais. Com isso, diversos tipos de estímulos podem ser transmitidos por dispositivos específicos e percebidos por um ou mais sentidos humanos (Burdea e Coiffet, 2003; Tori et al., 2006).

Três características são desejáveis em aplicações de RV: imersão, interação e presença. A imersão pode ser obtida pelo usuário por meio de dispositivos especiais 
como videocapacetes (head-mounted display - HMD) e rastreadores de posição. A interação refere-se à comunicação entre o usuário e o Ambiente Virtual (AV) $\mathrm{e}$, finalmente, a presença, neste contexto, refere-se à sensação do usuário sentir-se envolvido na aplicação, sendo participante dela. Dentre as três características citadas, a área de saúde requer especificidades críticas que devem ser consideradas.

Em relação à interação, observa-se que algumas aplicações, como o treinamento de procedimentos médicos, exigem o uso de dispositivos hápticos, ou seja, aqueles que proporcionam retorno tátil e de força a fim de provocar sensações relacionadas ao toque, necessárias à exigência de realismo.

A imersão também constitui um aspecto necessário em algumas aplicações, mas dispensável em outras. No ensino de anatomia por meio de atlas virtuais, por exemplo, o usuário não precisa se sentir dentro do $\mathrm{AV}$, mas esta característica pode torná-lo mais motivado ao aprendizado. No entanto, aplicações para o tratamento de fobias, como citado em Wauke et al. (2005) podem não apresentar o efeito desejado se não proporcionarem a sensação de imersão ao paciente.

A presença é fundamental para as aplicações de RV em saúde nas quais o usuário está fisicamente dentro do AV e deve participar dele (Costa et al., 2010). As aplicações de saúde, por excelência, demandam esta característica para que o usuário, de fato, sinta que os ambientes virtuais proporcionam experiências envolventes próximas às situações reais.

Em relação aos aspectos técnicos, conforme citado em Nunes e Costa (2008), para que o usuário perceba a sensação de presença em aplicações de saúde é fundamental que se estabeleçam alguns pré-requisitos:

- qualidade dos objetos tridimensionais os objetos que compõem o mundo virtual devem ser similares aos objetos reais em relação a cores, volumes, texturas, atividades e comportamentos;

- Correlação espacial entre objetos físicos e virtuais - as proporções de tamanho $\mathrm{e}$ localização de objetos no mundo virtual devem ser observadas a fim de que representem com propriedade o mundo real;

- Controle realista da interação - as ações nos AVs devem considerar o comportamento físico dos objetos e pessoas;

- Uso simultâneo de diversos dispositivos - a demanda de tempo de processamento e a definição dos limites de influência de cada dispositivo são pontos estratégicos que devem ser planejados tecnicamente;

- Aspectos ergonômicos - a modelagem de objetos e a inclusão de dispositivos não convencionais devem considerar a usabilidade da aplicação. Usabilidade aqui é compreendida como a característica que torna a aplicação confortável ao usuário.

\section{Aplicações de Realidade Virtual em saúde}

As aplicações de RV voltadas à saúde podem ser categorizadas de acordo com suas finalidades. Neste caso, destacam-se as aplicações para treinamento de procedimentos, educação, reabilitação, jogos, apoio ao desenvolvimento e sistemas colaborativos. Esta categorização pode ainda considerar o público-alvo, como apresentado na Figura 1.

A educação e o treinamento são os temas mais explorados no contexto das aplicações da RV para saúde. Aplicações dessas categorias visam a preparar estudantes, técnica e psicologicamente, para desenvolverem tarefas reais, auxiliar o processo educacional em diferentes faixas etárias, oferecer a profissionais meios de treinar novas técnicas simulando diferentes situações genéricas (desassociadas das peculiaridades de uma situação ideal), assim como facilitar o ensino de hábitos a pacientes. Neste contexto, encontram-se também os simuladores, cujo objetivo geral é permitir a prática de técnicas em ambientes que imitam a realidade de um procedimento ou criam situações hipotéticas para explicar ou ensinar algum conceito. Na maioria das vezes, sistemas dessa natureza fornecem visualização estereoscópica (visualização que propicia a sensação de profundidade nos AVs) e preocupam-se em oferecer algum tipo de retorno (tátil, visual ou auditivo) às interações do usuário. Os jogos também têm sido utilizados neste contexto, de forma a favorecer uma maior motivação para o aprendizado de conteúdos (Machado et al., 2011).

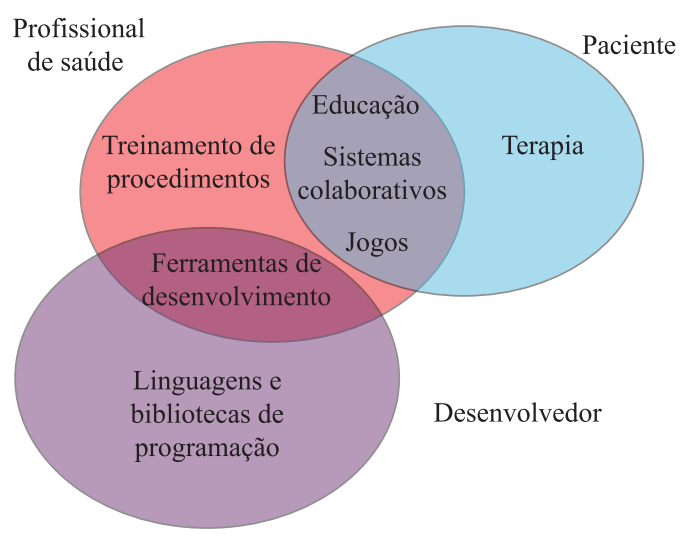

Figura 1. Categorias de aplicações de RV de acordo com o público-alvo. Figure 1. Categories of VR applications according to the target audience. 
A área de reabilitação tem sido abordada em sistemas de RV para treinar o paciente visando à recuperação de movimentos perdidos, bem como avaliar e reabilitar processos cognitivos como a percepção visual, a atenção e a memória (Parsons e Rizzo, 2008). Características desejáveis nesses sistemas são o realismo visual e a interação intuitiva (geralmente com dispositivos destinados a monitorar movimentos), que devem oferecer imersão, permitindo ao usuário concentrar-se na tarefa a ser desempenhada.

As aplicações colaborativas de RV tratam basicamente de situações em que a distância é um fator crítico. Seu objetivo é propiciar o contato e a troca de informações entre usuários com a sensação de presença mútua. Por esta razão, o conceito de presença é fundamental e permite a realização de atividades para obtenção de diagnósticos, segunda opinião e treinamento a distância, como exemplos.

Com o objetivo de facilitar o desenvolvimento de aplicações para a área de saúde, linguagens, bibliotecas e outras ferramentas com funcionalidades intrínsecas para este contexto, também têm sido objetos de pesquisa. Devido à facilidade de operação, algumas dessas ferramentas podem ser utilizadas por profissionais da saúde para construir aplicações simplificadas.

Aplicações de RV em telemedicina são incipientes devido, principalmente, ao tempo de transmissão de dados ainda ser alto. Destacam-se também trabalhos que abordam a apresentação gráfica em tempo real, detecção de colisão interativa, reconstrução volumétrica e desenvolvimento de dispositivos de exploração sensorial, dentre outros.

Em nível internacional, muitos grupos despontam e consolidam-se com publicações em diferentes revistas e em conferências, dentre as quais a Medicine Meets Virtual Reality (http://www.nextmed.com), realizada desde 1993 nos Estados Unidos e a International Conference \& Exhibition on Virtual Reality \& Converging Technologies (http://www. laval-virtual.org), que acontece na França desde 1999. A área de reabilitação conta com duas conferências que atualmente se alternam bianualmente: Virtual Rehabilitation International Conference que acontece desde 2003 (http://www.virtual-rehab.org) e a International Conference on Disability and Associated Technologies que começou em 1996 (http://www. icdvrat.reading.ac.uk). Entretanto, diversos trabalhos relacionados têm sido publicados em eventos de outras áreas, haja vista a inter e a muldisciplinaridade envolvidas nas aplicações de RV.

\section{Exemplos de aplicações no Brasil}

No Brasil, existem grupos de pesquisa distribuídos em todas as regiões do país que, em geral, vêm trabalhando especificamente na concepção de sistemas para educação, treinamento, reabilitação e ferramentas para o desenvolvimento rápido de tais aplicações. A seguir, são apresentados alguns sistemas de RV desenvolvidos por grupos de pesquisa brasileiros, objetivando exemplificar os diferentes usos da tecnologia de RV para a área de saúde, destacando as técnicas exploradas e os avanços que essas aplicações trazem para a área de saúde.

Na área de educação, o trabalho de Ramos e Nunes (2005) define um Atlas Virtual com o objetivo de facilitar o estudo de anatomia e fisiopatologia do câncer de mama. O sistema permite o acesso a uma base dados, fornecendo informações sobre as estruturas anatômicas, em tempo real durante a navegação do usuário, além de possibilitar a visualização do crescimento de tumores malignos (Figura 2). Neste caso, é propiciada uma inovação para o aprendizado uma vez que estruturas tridimensionais podem ser visualizadas e manipuladas de maneira similar ao mundo real. A navegação é realizada por meio de mouse e teclado e a estereoscopia é propiciada pela técnica de anaglifos.

Da mesma forma, o AnatomI 3D (Cunha et al., 2006) é uma estrutura de atlas digital de uso livre que permite, de forma interativa, a manipulação e o estudo de estruturas tridimensionais do corpo humano,

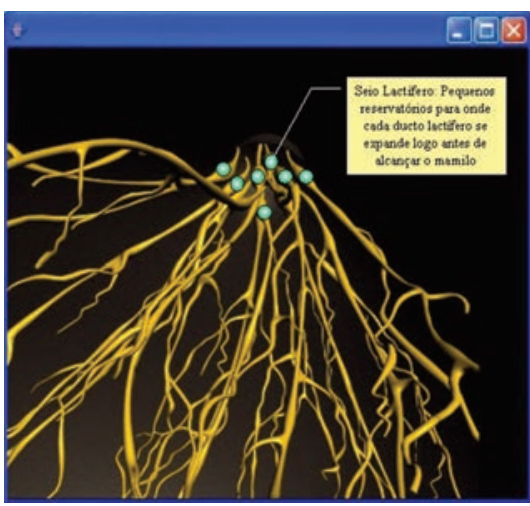

(a)
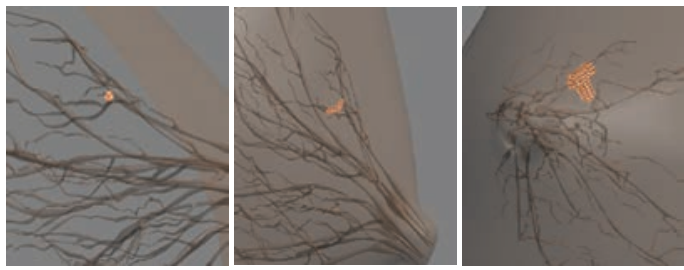

(b)

Figura 2. Atlas Virtual da Mama: a) Exibição de informações durante a navegação; b) Exemplo de desenvolvimento de um câncer em diferentes estágios (Ramos e Nunes, 2005).

Figure 2. Virtual Atlas of the Breast: a) Information exhibition during navigation, b) Example of a cancer development at different stages (Ramos e Nunes, 2005). 
associando de forma adaptativa textos descritivos sobre os modelos anatômicos (Figura 3). Desse modo, o usuário pode manipular as estruturas, inclusive suprimindo suas partes, ou observar a interconexão entre elas habilitando recursos de transparência e estereoscopia.

O projeto VIDA (Virtual Interactive DistanceLearning on Anatomy) é um Ambiente Virtual (AV) de aprendizagem, em desenvolvimento, baseado em atlas anatômico, que pode ser acessado a distância, via Internet, com o conceito de permitir o emprego de manipulação direta, isto é, o uso de partes do corpo em vez de movimentação e acionamento de botões de dispositivos (Tori et al., 2009). A Figura 4 apresenta uma ilustração conceitual da manipulação dos elementos do atlas virtual, conforme percebida pelo usuário. Uma ou mais webcams podem capturar o movimento das mãos e o sistema responde de acordo com a posição e a orientação dessas.

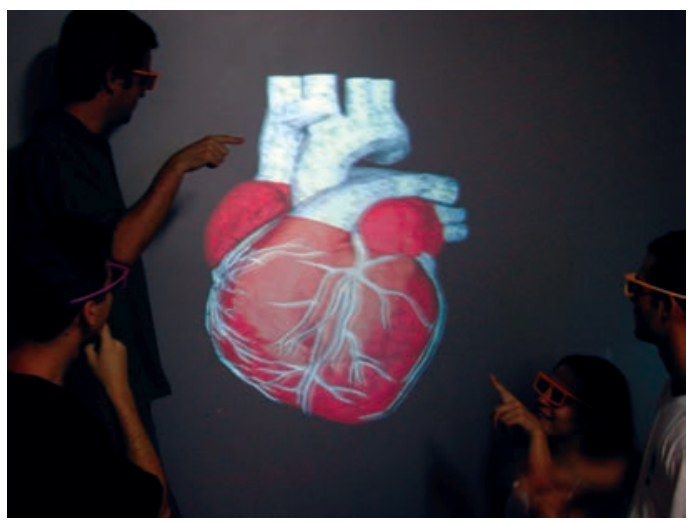

Figura 3. Exemplo de utilização do AnatomI 3D para visualização de estruturas do coração com estereoscopia (Cunha et al., 2006).

Figure 3. Example of the 3D AnatomI use for visualization of heart structures with stereoscopy (Cunha et al., 2006).
Considerando a área de treinamento de procedimentos, o primeiro simulador cirúrgico brasileiro utilizando RV visou a simulação do procedimento de coleta de medula óssea para transplante em crianças (Machado et al., 2002) (Figura 5). Este projeto contou com uma equipe multidisciplinar para integrar um sistema capaz de prover retorno tátil e de força, por meio de um dispositivo háptico, manipulação espacial e visualização estereoscópica durante o processo de coleta de medula óssea da região pélvica em um corpo virtual.

O SITEG - Sistema Interativo para Treinamento em Exames Ginecológicos é um simulador que tem como finalidade prover um ambiente virtual interativo, no qual o usuário pode aprender, treinar e ser avaliado sobre a realização de um exame ginecológico do colo do útero. Para tanto, o sistema apresenta diferentes patologias relacionadas ao colo do útero que podem ser identificadas em um exame ginecológico e conta com um módulo inteligente baseado em lógica fuzzy que monitora as opções e ações do usuário, classificando sua atuação. O procedimento virtual demanda o uso de um dispositivo háptico para simular o utensílio médico e prover ao usuário sensações associadas ao toque no corpo virtual (Machado e Moraes, 2006).

A simulação de exame de biópsia mamária foi explorada por Lima et al. (2004). O simulador desenvolvido disponibiliza objetos que representam uma mama e uma seringa virtuais, com interação por meio de mouse e teclado e detecção de colisão. Esta ferramenta deu origem a um framework, para gerar uma linha de produtos no domínio de exames de punção, citado adiante.

Na área de reabilitação, um grupo de pesquisadores da UFRJ e UERJ desenvolveu experimentos com ambientes virtuais tridimensionais para estimular a realização de atividades de vida diária de pessoas
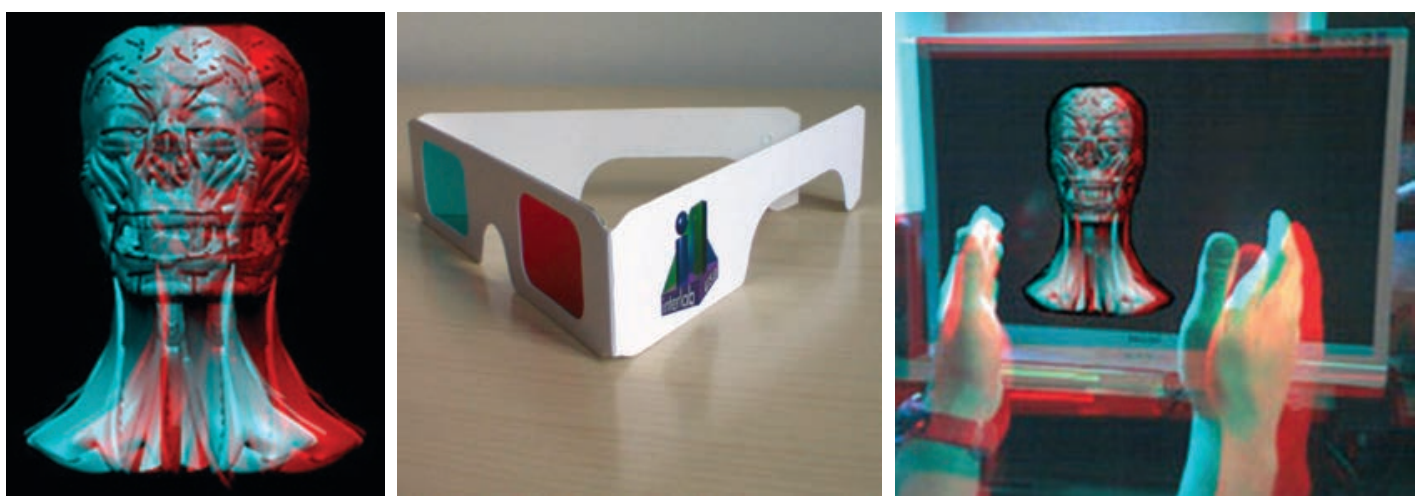

Figura 4. Projeto VIDA: modelo anatômico exibido em formato de estéreo anaglifo; par de óculos descartável necessário para visualização estéreo de imagens em formato de anaglifo e interação direta para manipulação do modelo tridimensional (Tori et al., 2009).

Figure 4. VIDA Project: anatomical model displayed in stereo anaglyph format; pair of disposable glasses needed for viewing images in stereo anaglyph format for direct interaction and manipulation of three-dimensional model (Tori et al., 2009). 
com sequelas de atenção e memória causadas por diferentes tipos de acidentes cerebrais e distúrbios neuropsiquiátricos. Nos experimentos desenvolvidos, os pacientes aceitaram com entusiasmo o trabalho nos ambientes virtuais e os resultados obtidos demonstraram uma significativa recuperação dos níveis de atenção e concentração nas atividades cotidianas (Cardoso et al., 2006; Costa e Carvalho, 2004). Foram utilizados o mouse e o monitor na primeira fase dos experimentos e o capacete de visualização 3D na segunda fase.

A constante demanda de intervenções do terapeuta durante a navegação dos pacientes nos ambientes virtuais estimulou a busca por novos meios de controle automático dos níveis de dificuldade das atividades propostas. Neste sentido, técnicas de Inteligência Artificial vêm sendo integradas nos ambientes, de modo a realizar a alteração dos níveis de complexidade das tarefas, sem a necessidade de atuação dos profissionais que estão responsáveis por acompanhar o processo terapêutico (Costa et al., 2010).

Como consequência relacionada às múltiplas demandas de hardware e software, bem como sua compatibilização durante o desenvolvimento de aplicações de simulação de procedimentos para RV, foi proposto em (Oliveira e Nunes, 2010) o framework ViMeT (Virtual Medical Training), que disponibiliza um conjunto de classes em Java para construção de aplicações para simulação de exames de biópsia. Atualmente, o ViMeT disponibiliza pacotes de código para implementar um ambiente virtual com detecção de colisão com precisão, deformação, estereoscopia e inclusão de dispositivos não convencionais (Corrêa et al., 2009). Para facilitar a geração automatizada de ferramentas foi disponibilizada uma ferramenta Wizard que, a partir de parâmetros fornecidos pelo usuário, gera uma aplicação que pode ser customizada pelo programador (Nunes et al., 2007). A Figura 6 apresenta aplicações geradas com o framework ViMeT.

No ano de 2007 foi disponibilizado livremente o framework CyberMed, com foco específico no desenvolvimento de aplicações para treinamento médico baseadas em RV (Machado et al., 2009). O framework oferece suporte a tarefas de visualização estereoscópica interativa, uso de dispositivos não convencionais, deformação interativa, rastreamento, tarefas colaborativas e métodos para avaliação online do usuário (Figura 7). $\mathrm{O}$ projeto permanece ativo e disponível em http://cybermed.sourceforge.net.

Uma área que vem sendo explorada recentemente é a definição de metodologias de avaliação do usuário em treinamento. Apesar de que os primeiros trabalhos nessa área datarem do início dos anos 90 , só a partir dos

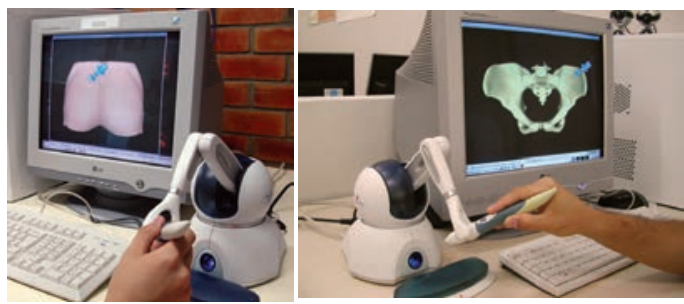

Figura 5. Simulador de coleta de medula óssea (Machado et al., 2002) Figure 5. Simulator for Bone Marrow Harvest (Machado et al., 2002).
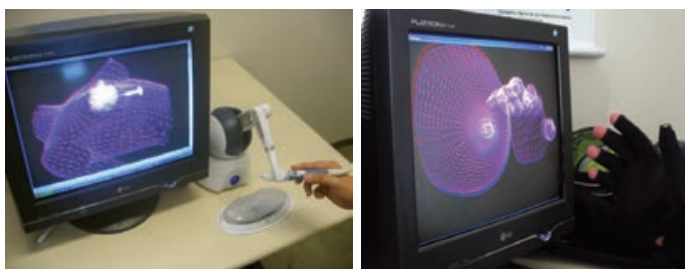

Figura 6. Aplicações geradas com o framework ViMeT utilizando dispositivos não convencionais - equipamento háptico e luva de dados (Corrêa et al., 2009).

Figure 6. Applications generated with the ViMeT framework using non-conventional devices - haptics equipment and dataglove (Corrêa et al., 2009).

anos 2000 é que essa área realmente vem despertando interesse (Moraes e Machado, 2009, 2011). Neste contexto, vários autores têm apresentado técnicas para avaliação do usuário em treinamento com o objetivo de monitorar suas ações e classificar suas habilidades utilizando diferentes métodos para vários propósitos (Chang, 2008; Machado e Moraes, 2010; Wang et al., 2010). O mesmo vem sendo realizado para múltiplos usuários em treinamento interagindo em um mesmo ambiente virtual, remotamente ou não (Moraes e Machado, 2007). Também merecem destaque os trabalhos, ainda em fase inicial de (Santos et al., 2010) e (Obana e Tori, 2010), que visam a definir metodologias para avaliar, respectivamente, o nível de cognição e o nível de presença percebida pelos usuários em ambientes virtuais tridimensionais. Esses trabalhos têm sido publicados, em sua maioria, em eventos nacionais que integram as áreas tecnológicas e da saúde.

Visando a obter um panorama dos trabalhos que abordam aspectos da tecnologia de RV, a seguir são apresentados os resultados de uma pesquisa que visou obter subsídios para discutir as necessidades e oportunidades da área.

\section{Materiais e Métodos}

No Brasil, poucos periódicos contemplam temas relacionados à RV aplicada à saúde. Entretanto, alguns eventos já consolidados publicam trabalhos dos diferentes grupos de pesquisa brasileiros que se 


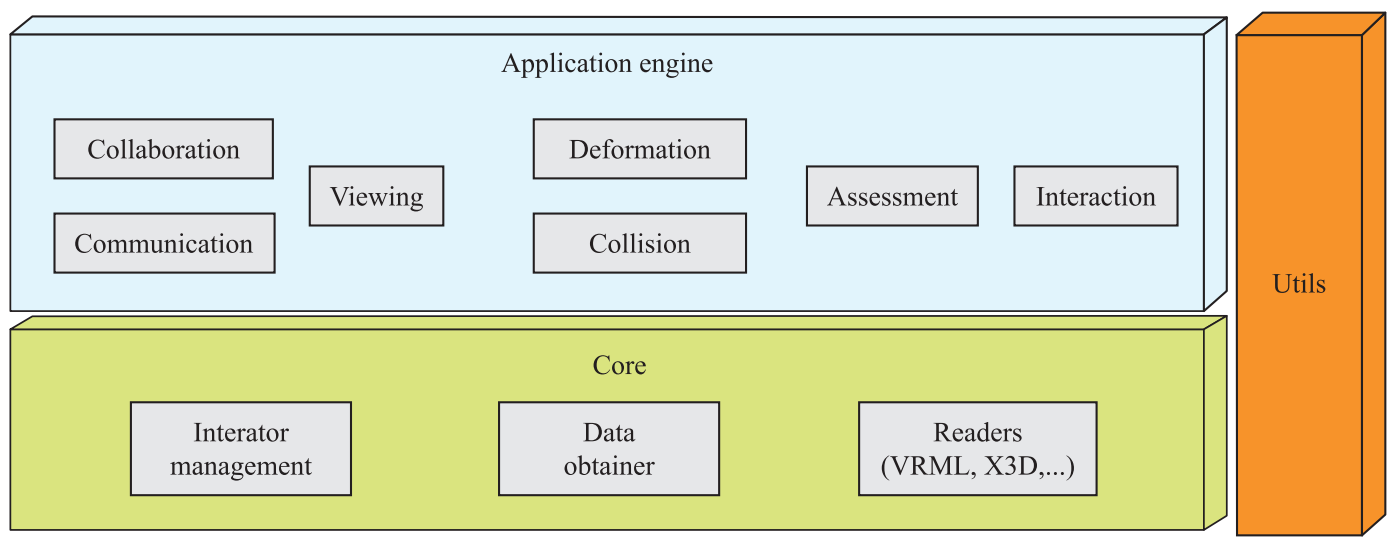

Figura 7. Componentes do framework CyberMed.

Figure 7. Components of the CyberMed framework.

dedicam a este assunto. De maneira a se obter um panorama da área de RV aplicada às áreas da saúde no país, a seguir são apresentados e discutidos os dados de uma pesquisa realizada em veículos nacionais de divulgação científica.

Para a presente pesquisa foram selecionados os veículos de divulgação científica que mais se enquadram no contexto do presente artigo. No caso de conferências, foram selecionadas as edições mais recentes, considerando pelo menos os últimos 5 anos disponíveis do Congresso Brasileiro de Engenharia Biomédica-CBEB (2004 a 2009), do Workshop de Informática Médica-WIM (2004 a 2010), do Symposium on Virtual and Augmented Reality-SVR (2006 a 2010) e do Congresso Brasileiro de Informática em Saúde-CBIS (2004 a 2010). Também foi analisada a Revista Brasileira de Engenharia Biomédica (1999 a 2010) por ser o periódico mais consolidado da área analisada (outros estão em fases iniciais no Brasil ou com edições insuficientes para análise). A intenção foi verificar a quantidade e as características das aplicações de RV na área da saúde desenvolvidas no país a fim de tecer uma visão da situação nacional a respeito das pesquisas na área.

Para o levantamento dos dados foi realizada uma análise detalhada dos trabalhos publicados nos veículos selecionados. Os trabalhos que se relacionavam com a área de interesse foram classificados de acordo com o tipo da aplicação, as áreas da saúde, o ano, o veículo de publicação, os tipos de dispositivos utilizados e existência de avaliação ou validação do software. Os dados foram agrupados de modo a representar graficamente os resultados e permitir que as diferentes situações e tendências desta área pudessem ser analisadas.

\section{Resultados}

A partir do estudo conduzido foram obtidos dados da evolução quantitativa dos trabalhos publicados, conforme ilustrado na Figura 8, totalizando 38 trabalhos. É possível perceber um tímido aumento de trabalhos publicados em eventos no período de 2005 a 2007 em relação ao ano de 2004.

Embora haja trabalhos de RV na área de saúde em todos os eventos selecionados, a pesquisa na Revista Brasileira de Engenharia Biomédica (RBEB) indicou que trabalhos da área não foram publicados neste periódico no período de 2004 a 2010. Por essa razão, tal veículo não foi incluído nos resultados apresentados a seguir.

Na Figura 9 é apresentada a distribuição dos trabalhos de acordo com a área de aplicação. As categorias "Apresentação de conteúdos” e "Treinamento de procedimento" estão relacionadas com processos educativos. Entretanto, a primeira visa a permitir a visualização e a interação com objetos $3 \mathrm{D}$ de forma exploratória enquanto a segunda visa à prática de tarefas com a finalidade de aquisição de habilidades. Para essas categorias, o público-alvo típico é constituído de estudantes e profissionais da área de saúde. As aplicações classificadas como "Ferramenta de desenvolvimento" dizem respeito a componentes de software para criação de aplicações, visando ao aumento de produtividade por meio do reuso de código.

Na Figura 10 observa-se a distribuição do uso de dispositivos nas aplicações publicadas, salientado que cada uma delas pode ter envolvido mais de um tipo de dispositivo. Observa-se que apesar da existência de dispositivos não convencionais em várias delas, a maioria ainda contempla somente dispositivos convencionais como teclado e mouse. 


\section{Evolução quantitativa}

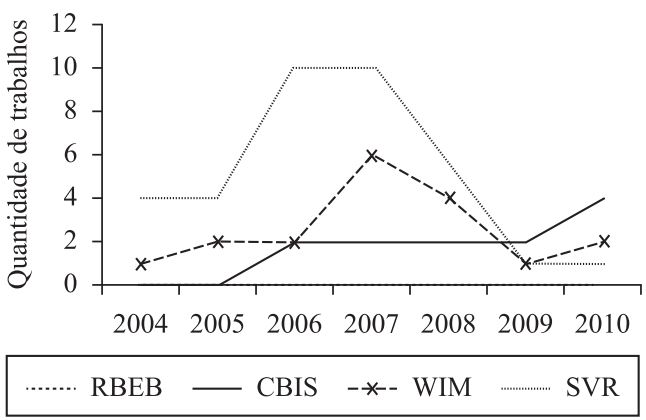

Figura 8. Evolução quantitativa dos trabalhos de RV em saúde publicados entre os anos de 2004 e 2010.

Figure 8. Quantitative evolution of the works of VR for health care published from 2004 to 2010.

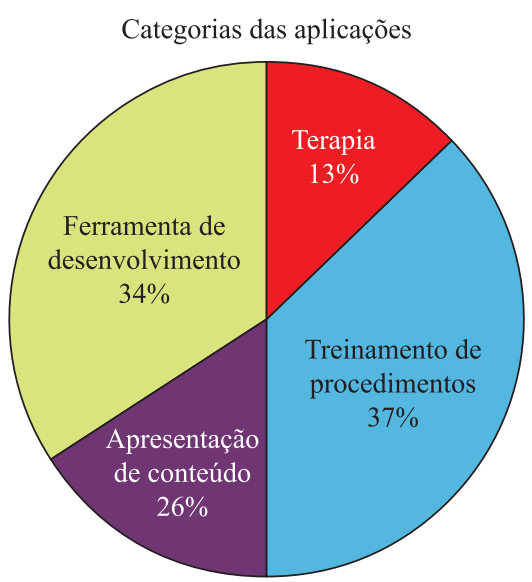

Figura 9. Distribuição dos trabalhos publicados de acordo com as áreas de aplicação.

Figure 9. Distribution of published papers according to application areas.

Uso de dispositivos

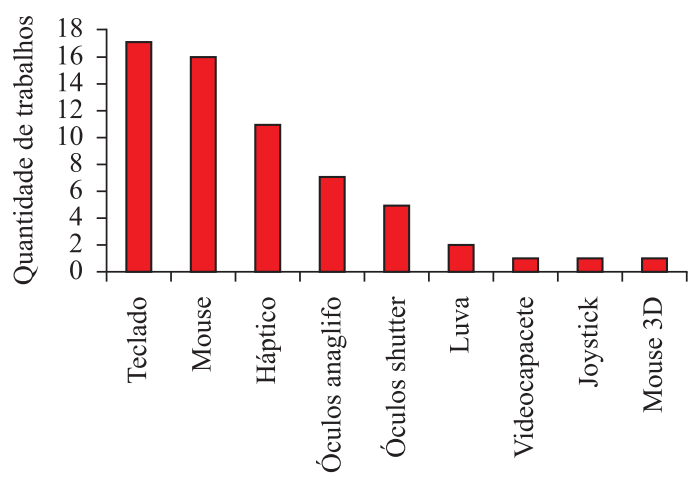

Figura 10. Uso de dispositivos nos trabalhos pesquisados. Figure 10. Use of devices in the investigate works.
A existência de processos de avaliação para as aplicações é apresentada na Figura 11. Esses processos relacionam-se a algum tipo de atividade avaliativa do software, considerando o usuário final, os requisitos técnicos ou a eficácia. Do universo dos trabalhos que citaram algum tipo de avaliação (45\% do total), verifica-se que nenhum artigo incluiu todos esses três aspectos. Na maioria dessas publicações foram apresentadas apenas avaliações com o usuário no final do processo de desenvolvimento e com a finalidade de verificar se as funcionalidades atendem aos requisitos delineados. Em alguns artigos são citadas avaliações técnicas, geralmente sendo mensurada a quantidade média de quadros por segundo, obtida durante a geração gráfica da cena tridimensional. Nenhum dos artigos cita avaliações executadas durante todo o processo de desenvolvimento.

\section{Discussão}

Na Figura 8 é possível observar que em dois veículos houve um aumento no número de trabalhos entre 2005 e 2007 e, depois deste período, a quantidade de trabalhos publicados diminuiu. Especular a causa desta diminuição é tarefa arriscada, visto que o decremento da quantidade de publicações não significa necessariamente a diminuição de pesquisas na área. Entretanto, observa-se que a partir deste período muitos pesquisadores intensificaram a busca por publicações internacionais devido a exigências de programas de pós-graduação no país. Esta tendência pode ser observada nos artigos publicados na revista Scientometrics (Wainer et al., 2009), que corroboram a percepção do aumento de publicações internacionais dos pesquisadores brasileiros nos últimos anos, o que pode ter causado a diminuição da quantidade de publicações da área no país. Também confirma esta tendência o relatório da Unesco sobre o desenvolvimento da ciência no mundo, que mostra um aumento de $110,6 \%$ na produção científica brasileira em periódicos indexados de 2008 quando comparada à produção de 2002 (Unesco, 2010).

Como mencionado, muitas aplicações desenvolvidas no Brasil são relacionadas ao treinamento de procedimentos, como pode ser conferido na Figura 9. Considerando esta área e a apresentação de conteúdos, pode-se verificar que aplicações com foco educativo somam $53 \%$ das iniciativas de pesquisa. As necessidades da área de saúde em relação a novos métodos de treinamento, novos materiais, diminuição de custos e disponibilidade de recursos, certamente são os principais propulsores dessas ações. De fato, a literatura destaca que o uso de ferramentas virtuais para a educação em saúde tem potencial para diminuir a necessidade de laboratórios físicos (Ma 


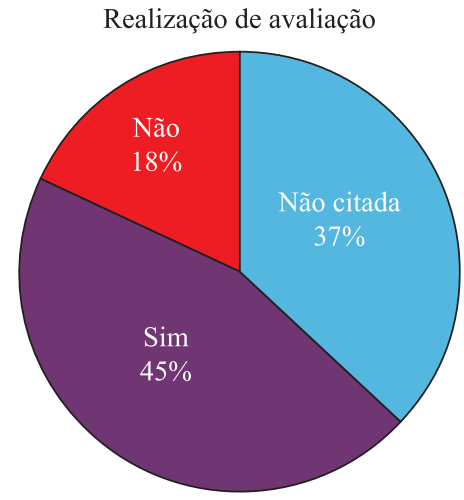

Figura 11. Realização de avaliação nos trabalhos pesquisados. Figure 11. Conduction of evaluation in the investigated papers.

e Nickerson, 2006) e propiciam a transferência de conhecimento das situações treinadas virtualmente para ambientes reais (Johnsen et al., 2007).

Apesar de aparentemente a área de treinamento de procedimentos ser predominante quando as aplicações são categorizadas considerando-se as suas finalidades, salienta-se que vários tipos de procedimentos são pouco contemplados pelos grupos brasileiros. Em geral, as aplicações desenvolvidas contemplam procedimentos na área de Medicina e outras áreas da saúde - tais como Odontologia e Fisioterapia são pouco lembradas. Isso também acontece com as aplicações da área de terapia: essa categoria inclui majoritariamente aplicações para as áreas de Psicologia e Psiquiatria, sendo que há carência de aplicações de RV aplicada, por exemplo, à reabilitação e ao planejamento de terapias considerando características específicas de pacientes.

A partir das informações apresentadas na Figura 10, percebe-se que a maioria das aplicações ainda utiliza dispositivos convencionais (mouse e teclado), levando a acreditar que o preço de dispositivos não convencionais ainda é um fator limitante para o desenvolvimento e/ou uso de sistemas de RV para a área de saúde. A utilização de equipamentos hápticos apresenta um crescimento recente, justamente devido ao desenvolvimento de aplicações direcionadas ao treinamento de procedimentos que exige a percepção, por parte do usuário, das propriedades físicas (como resistência, textura e elasticidade) dos componentes do corpo humano, como órgãos e tecidos, representados por objetos virtuais 3D. Também é possível observar por meio do gráfico apresentado na Figura 10 que dispositivos para aumentar o grau de imersão, como óculos para visualização estereoscópica (anaglifo ou shutter), assim como videocapacetes (HMD), têm sido contemplados nas aplicações. Apesar de existirem dispositivos de visualização 3D ainda com alto custo, técnicas de estereoscopia, como a de anaglifos, também favorecem a imersão mantendo o desenvolvimento de projetos de pesquisa com custo baixo. Exemplos de óculos que proporcionam visualização estereoscópica, luvas de dados e dispositivos hápticos foram apresentados nas Figuras 4-7. Na Figura 13 são apresentados exemplos de videocapacetes.

Alguns grupos no Brasil desenvolvem ferramentas destinadas a auxiliar na produção de aplicações, como pode ser observado na Figura 9. Tais ferramentas, em geral disponibilizadas no formato de frameworks, possibilitam o aumento da produtividade no desenvolvimento de software, pois fornecem pacotes de código que podem ser reutilizados para implementar funcionalidades comuns em aplicações para a área de saúde. Exemplos dessas funcionalidades são a visualização tridimensional com técnicas variadas, a manipulação interativa de objetos deformáveis e táteis, técnicas de concorrência e suporte ao uso de dispositivos não convencionais, dentre outras.

Um ponto crítico observado nas aplicações publicadas no contexto analisado é a ausência de avaliações, tanto em relação à usabilidade dos sistemas, quanto em relação à sua eficácia e à consonância dos requisitos funcionais para os quais a ferramenta foi concebida. Como pode ser observado no gráfico da Figura 11, apenas $45 \%$ dos trabalhos publicados apresentaram algum tipo de avaliação. Esta característica é uma fragilidade que influencia diretamente na utilização dessas ferramentas. Este aspecto deve ser explorado e melhorado a fim de que esses sistemas possam ser validados e incluídos na rotina de trabalho dos profissionais de saúde. Diferentemente de aplicações de RV para outras áreas, aplicações para a área de saúde exigem um alto grau de eficácia, pois erros podem colocar em risco a saúde dos pacientes.

Este quadro delineia uma área de pesquisa a ser explorada no contexto de RV, visto que ainda não se encontram na literatura metodologias de avaliação consolidadas que permitam verificar o desempenho das ferramentas desenvolvidas, considerando aspectos técnicos, éticos, cognitivos e sensório-motores, dentre outros. Também não são encontradas na literatura metodologias desenvolvidas para que os sistemas de $\mathrm{RV}$ possam ser avaliados com usuários reais quando esses são efetivamente pacientes submetidos a sessões de terapia que incluem ambientes virtuais. Destaca-se que, especificamente no caso de pacientes reais, os experimentos devem incluir precauções adicionais, visto que alguns dispositivos de RV podem causar mal-estar - como enjoos e vertigens, dependendo da sensibilidade do paciente. Por isso, é essencial o 
consentimento do paciente ou do seu responsável e a aprovação do Comitê de Ética em Saúde Humana, garantindo, assim, a preservação da integridade do paciente.

Um desafio adicional a ser apontado em relação à avaliação desses sistemas com usuários reais é a conhecida distância entre a academia e a indústria (no caso, as instituições de saúde). Com exceção de instituições públicas diretamente ligadas a centros de pesquisa (principalmente universidades), no Brasil esta colaboração ainda é incipiente. Alguns fatores que contribuem para aumentar ou, no mínimo, manter tal espaço são a diferença entre linguagens, o tempo de resposta e a natureza dos interesses finais. Muitas vezes o setor produtivo está interessado em produtos acabados ou na obtenção de patentes em períodos de tempo curtos e a academia preocupa-se com a geração do conhecimento e a produção intelectual, expressa principalmente por publicações de artigos. Enquanto a indústria no Brasil ainda não investir massivamente em pesquisa, inclusive absorvendo mão-de-obra qualificada para desenvolvê-la, uma iniciativa que pode auxiliar na diminuição desta dicotomia é o

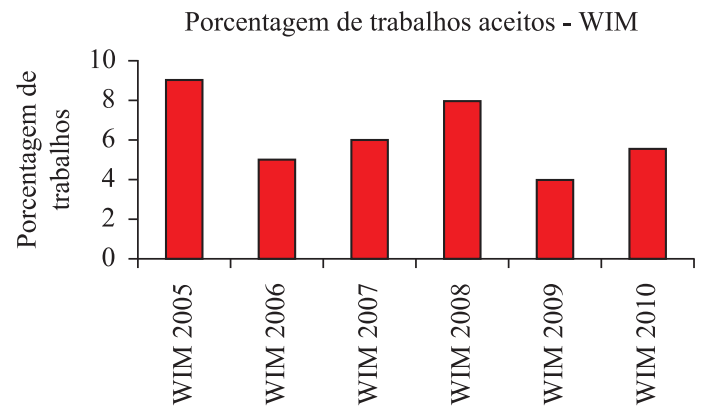

Figura 12. Porcentagens de trabalhos do WIM contemplando Realidade Virtual.

Figure 12. Percentages of works published in the WIM conference covering Virtual Reality. lançamento de editais de financiamento que possuam incentivo para parceria academia-indústria (o que já vem acontecendo - ainda que timidamente - em alguns órgãos de fomento).

Outra indicação interessante a partir desses resultados é que a quantidade de trabalhos de RV em saúde, nos eventos pesquisados, ainda é bem pequena quando comparada à quantidade de outras áreas da Computação aplicada à saúde. A título de ilustração, a Figura 12 apresenta as porcentagens de trabalhos de RV publicados no WIM, no período de 2005 a 2010 divididos por área da Computação. Verifica-se, no caso específico deste evento, que a porcentagem máxima de trabalhos de RV publicados foi $9 \%$ em 2005, indicando que há, ainda, muito espaço para pesquisa e desenvolvimento usando essa tecnologia.

A parceria entre a tecnologia de RV e a área de saúde apresenta limitações e desafios a serem vencidos dos dois lados. Neste sentido, observou-se que a maioria dos trabalhos pesquisados não possui participação do setor produtivo. Exemplificando, todos os trabalhos publicados no SVR no período consultado são oriundos da academia, não apresentam qualquer participação da indústria, embora vários possuam financiamento governamental. Provavelmente este é um dos motivos pelos quais os trabalhos classificados na categoria "Ferramenta de desenvolvimento" (Figura 9) concentram-se na concepção de software livre ou aberto. Este paradigma também evidencia a preocupação que alguns grupos de pesquisa têm em disponibilizar meios pelos quais a comunidade possa desenvolver novas aplicações minimizando o volume de recursos necessários para a aquisição de licenças de software, visto que desenvolver aplicações imersivas, conforme já foi observado, pode envolver alto custo devido à necessidade de dispositivos não convencionais. Assim, a disponibilização de bibliotecas
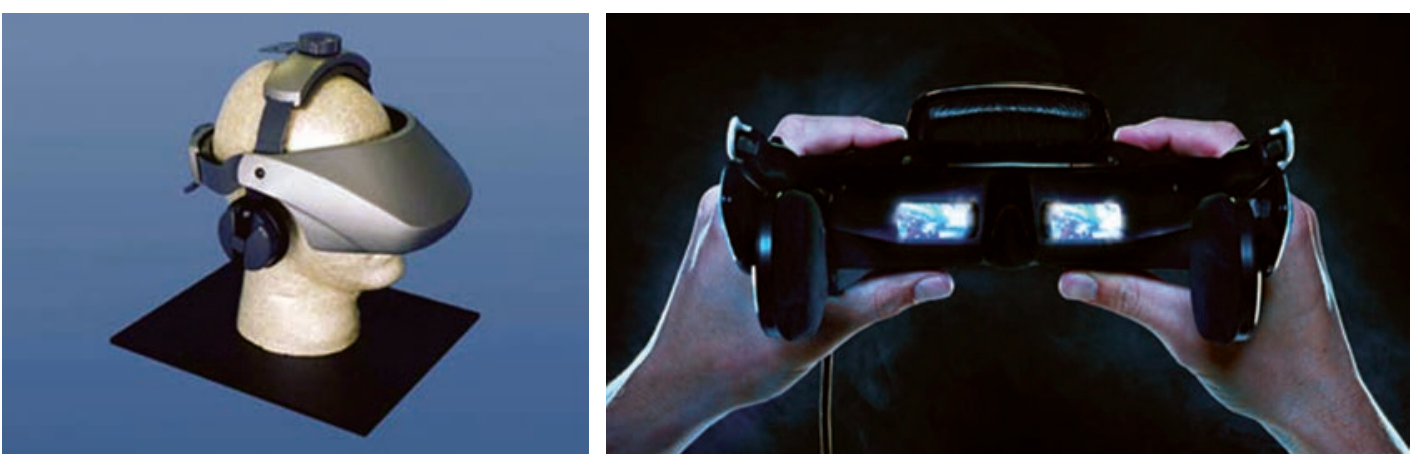

Figura 13. Exemplos de videocapacete usados em aplicações de RV (http://www.5dt.com/products/phmd.html - citado em 01 out 2011; http://www.sony.pt/hub/oculos-video-hmd - citado em 01 out 2011).

Figure 13. Examples of HMD (head-mounted display) used in VR applications (http://www.5dt.com/products/phmd.html - cited 2011 Oct 01, http://www.sony.pt/hub/oculos-video-hmd - cited 2011 Oct 01). 
e ferramentas de desenvolvimento gratuitas torna-se uma importante contribuição para a área.

Desenvolver aplicações de RV para a saúde exige atenção para diversos requisitos que devem ser atendidos. Velocidade, precisão, capacidade de armazenamento e adequação ergonômica de hardware são exemplos de desafios a serem vencidos na parte tecnológica. No entanto, nem sempre todos os requisitos precisam ser contemplados para atingir os objetivos almejados em uma aplicação. Cabe à equipe multidisciplinar estabelecer as prioridades e fazer escolhas que conduzam ao melhor resultado, respeitando limitações teóricas, técnicas e principalmente, as orçamentárias. A ausência dessa multidisciplinaridade nas equipes muitas vezes pode levar a deduções super estimadas quanto às necessidades reais da aplicação. Da mesma forma, isso pode conduzir à geração de aplicações equivocadas que não apresenta utilidade prática para o profissional de saúde.

O conhecimento do funcionamento da tecnologia, mudanças na educação dos profissionais para minimizar resistências e criação de espaços adequados para a utilização da tecnologia de RV em clínicas, hospitais e escolas constituem pontos a serem superados no lado da saúde. Além disso, o estabelecimento de uma linguagem comum para facilitar a comunicação e proporcionar a definição eficiente de requisitos é um desafio que ainda deve ser vencido pelas duas partes.

Em geral, as aplicações de RV na saúde descritas na literatura não chegam a ser produtos comerciais, nem ao menos chegam a ser usadas plenamente nos ambientes hospitalares ou em cursos da área da saúde. Este quadro parece estar diretamente ligado ao fato de poucos trabalhos relatarem resultados de testes de validação, fato que pode ter sido causado pela ausência de envolvimento efetivo de profissionais da saúde e do setor produtivo no processo. Isso resulta em um ciclo vicioso: não se usa porque não é testado e não é testado porque não há envolvimento desses setores. Em geral, ainda há pouca participação das indústrias nas universidades brasileiras (Cunha e Schenini, 2007), o que dificulta a criação de produtos comerciais com potencial de absorção pelo mercado. Apesar de haver tradição em outros países de apoio a pesquisas por empresas, este suporte financeiro está diminuindo (Dorsey et al., 2010). A combinação desses dois fatos poderá gerar impactos negativos no Brasil, fazendo com que o apoio financeiro da indústria diminua, o que tornaria mais frágil a ligação entre as empresas e as universidade.

No Brasil, a pesquisa na área é desenvolvida, sobretudo, em centros de pós-graduação, o que muitas vezes inviabiliza o recrutamento e o envolvimento de desenvolvedores em prazos longos, causando a lentidão no desenvolvimento dos projetos. Soma-se a isso o fato de que o investimento em pesquisa é, quase na sua totalidade, proveniente da iniciativa governamental e a escassez de verbas faz com que projetos iniciados com sucesso sejam descontinuados. Verifica-se que nos últimos anos o investimento em pesquisa no Brasil não acompanhou a evolução do Produto Interno Bruto (PIB), o que indica que o setor não tem recebido a atenção necessária para propiciar um real desenvolvimento ao país, conforme analisado no mais recente relatório da Unesco que analisa a situação da ciência no mundo (Unesco, 2010). Outra observação importante deste mesmo relatório é que de 2002 a 2007 a quantidade de pesquisadores no Brasil cresceu de 71,8 mil para 122,8 mil, mas o investimento em pesquisa não seguiu a mesma evolução, visto que a taxa de investimento em pesquisa caiu de US\$ 181,4 mil para US\$ 162,1 mil (Unesco, 2010).

É interessante ressaltar que nem sempre os dispositivos específicos para aplicações de RV hoje existentes são suficientes para o desenvolvimento de aplicações que atendam às necessidades dos profissionais de saúde. A partir da avaliação de algumas aplicações de treinamento médico para simulação de exames de biópsia realizada com profissionais da área (Corrêa et al., 2009), duas limitações da maioria das aplicações são observadas: a necessidade de manutenção de objetos físicos reais em alguns treinamentos e a inadequação dos dispositivos tangíveis. Em relação à primeira delas, isto poderia eventualmente ser minimizado com o desenvolvimento de sistemas de simulação híbridos, nos quais objetos físicos semelhantes àqueles utilizados no treinamento tradicional são empregados para facilitar aos usuários a correlação espacial com objetos virtuais utilizados na simulação.

Uma observação importante é que há poucos dispositivos tangíveis que proporcionam a sensação tátil adequada e, ao mesmo, sejam ergonomicamente semelhantes aos utilizados na prática dos procedimentos reais. Apesar de já existir uma gama de dispositivos hápticos comercialmente disponíveis, a forma, o tamanho e o peso dos mesmos não condizem com o necessário para simular adequadamente os equipamentos reais. Adicionalmente, dispositivos hápticos capazes de simular múltiplos pontos de contato apresentam limitações nas aplicações de tempo-real, tanto em custo quanto em utilização em sistemas que abordam múltiplas tarefas, como o caso dos sistemas de RV. Por esta razão, a simulação do contato físico em ambientes virtuais para a saúde ainda é limitada e concentra-se em atividades em que um único ponto de contato é suficiente, como cortes (simulação de 
bisturis) e perfurações (simulação de agulhas). Nestes casos, a detecção de colisões e reações advindas do contato exigem um processamento simultâneo com outras tarefas do sistema.

Apesar de o desenvolvimento das redes, do aumento da velocidade de comunicação e da capacidade de armazenamento e da ampliação da difusão de equipamentos de interação, poucos trabalhos consideram a possibilidade de desenvolverem sistemas disponíveis a distância, ou seja, sistemas que permitam atividades colaborativas entre profissionais da saúde. No Brasil, isso seria particularmente relevante dada à dimensão continental do país.

Por fim, é fundamental a observação dos aspectos éticos envolvidos em cada uma das aplicações, o que demanda disponibilidade de meios de controle por parte dos profissionais da saúde, que seriam responsáveis por gerenciar o uso e os resultados obtidos. O avanço dos preceitos de bioética tem gerado impactos em vários domínios das ciências médicas e perpassa, de maneira incisiva, o uso das novas tecnologias nesta área. Neste sentido, a utilização e a construção de artefatos tecnológicos devem receber especial atenção de maneira a não colocar em risco a saúde de seus usuários.

\section{Conclusões}

Considerando as características, o estado da arte e as limitações das aplicações de RV em saúde, as conclusões tecidas a seguir abordam uma série de oportunidades de pesquisa e desenvolvimento na área a fim de que os desafios sejam superados.

Como o desenvolvimento de aplicações de RV para a área médica envolve questões tecnológicas complexas, um primeiro aspecto que necessita receber a atenção de pesquisadores da área de Computação é o desenvolvimento de software (pacotes, bibliotecas, frameworks) que permitam a criação mais rápida de aplicações por meio de reutilização de código. Relacionado a este contexto, é também necessária a criação de bibliotecas de objetos sintéticos tridimensionais com nível de realismo para sua inclusão nas aplicações. Estes softwares devem possibilitar o desenvolvimento de aplicações de baixo custo (e, se possível, em software aberto), mas, com qualidade suficiente para proporcionar precisão nos procedimentos e resposta em tempo-real adequada.

Um segundo ponto importante é o desenvolvimento de hardware, com o objetivo de simular equipamentos utilizados no mundo real de forma mais intuitiva, ergonômica e precisa. O software básico relacionado ao hardware também constitui uma área desafiadora, pois precisa ser portável para as necessidades das aplicações.
Outro ponto ainda pouco explorado diz respeito ao estabelecimento de metodologia de avaliação dos sistemas. Para que as aplicações possam ser efetivamente incluídas na rotina dos profissionais da saúde, métodos de avaliação bem definidos devem ser desenvolvidos a fim de possibilitar a mensuração da adequação das ferramentas aos requisitos do usuário, à necessidade técnica e ao aprendizado, garantindo, assim, a exequibilidade e a assertividade das mesmas.

O uso da RV para fins de treinamento evidencia a necessidade de avaliar as ações do usuário, identificando falhas, dificuldades e acertos que permitam observar seu desempenho e seu progresso ao longo do treinamento, bem como a reconfiguração das tarefas das aplicações, em tempo real. Atualmente, a pesquisa de formas de avaliação online e de avaliação continuada de usuários em ambientes virtuais é uma importante área que permitirá no futuro utilizar, de forma mais abrangente, os sistemas de RV como ferramentas efetivas de treinamento na área médica (Machado e Moraes, 2010).

Profissionais, clínicas, hospitais, escolas e outras instituições da área de saúde podem ter na tecnologia de RV uma resposta às suas necessidades. Assim, a repetição de treinamento de procedimentos sem perigo a pacientes; a diminuição do uso de cadáveres, cobaias e materiais de manutenção e a simulação de situações antes somente imaginadas, como a visualização do funcionamento de um órgão humano ou a trajetória de um medicamento no organismo, constituem exemplos de aplicações que têm proporcionado o crescimento de um rico campo interdisciplinar de pesquisa e desenvolvimento.

A diminuição no preço e o aumento da capacidade de processamento dos equipamentos envolvidos nas tecnologias de RV podem ter sido fatores de motivação para a fixação de grupos de pesquisas na área, pois várias pesquisas significativas não exigem equipamentos caros. Entretanto, como novos desafios surgem em fluxo contínuo, o crescimento no número de grupos de pesquisa associado à difusão das práticas de controle da qualidade de processos e produtos contribuirão para aumentar o número de produtos de qualidade disponíveis no mercado e ampliarão a visibilidade das tecnologias de RV na área da saúde.

No Brasil há, ainda, várias barreiras a serem transpostas para que as aplicações eu empregam Realidade Virtual sejam efetivamente inseridas na rotina dos profissionais da área de saúde. Se por um lado elas constituem obstáculos para aproximar os profissionais dessa tecnologia, por outro lado tornam-se oportunidades ímpares de pesquisa inter e multidisciplinar, que podem contribuir sobremaneira com o desenvolvimento científico e tecnológico do país. 


\section{Agradecimentos}

A pesquisa aqui apresentada está inserida no contexto do Instituto Nacional de Ciência e Tecnologia de Medicina Assistida por Computação Científica (INCTMACC). Os autores agradecem ao Conselho Nacional de Desenvolvimento Científico e Tecnológico (CNPq), à Coordenação de Aperfeiçoamento de Pessoal de Nível Superior (CAPES), à Financiadora de Estudo e Projetos (Finep), à Fundação de Amparo à Pesquisa do Estado de São Paulo (Fapesp) e à Fundação de Amparo à Pesquisa do Estado do Rio de Janeiro (Faperj), pelo financiamento de projetos relacionados ao conteúdo deste artigo.

\section{Referências}

Brown DJ, Kerr S, Wilson JR. Virtual environments in special-needs education. Communications of the ACM. 1997; 40(8):72-5. http://dx.doi.org/10.1145/257874.257891

Butler DP, Willett K. Wii-habilitation: Is there a role in trauma? Injury. 2010; 41(9):883-5. PMid:20398899. http://dx.doi.org/10.1016/j.injury.2010.03.024

Burdea G, Coiffet P. Virtual reality technology. 2th ed. New York: John Wiley \& Sons; 2003.

Cardoso L, Costa RM, Piovesana A, Costa M, Penna L. Using virtual environments for stroke rehabilitation. In: International Workshop on Virtual Rehabilitation: Proceedings of the IEEE 5th International Workshop on Virtual Rehabilitation; 2006; New York. New York: IEEE; 2006. p. 1-5.

Chang T-C. Cumulative sum schemes for surgical performance monitoring. Journal of Royal Statistical Society: Series A. 2008; 171(2):407-32. http://dx.doi.org/10.1111/j.1467985X.2007.00513.x

Chestnut JA, Crumpton LL. Virtual Reality: a training tool in the 21st century for disabled persons and medical students. In: Biomedical Engineering Conference: Proceedings of the Biomedical Engineering Conference; 1997 Apr.; New Orleans. New Orleans; 1997. p. 418-21.

Chodos D, Stroulia E, Boechler P, King S, Kuras P, Carbonaro M, Jong E. Healthcare education with virtualworld simulations. In: 2010 ICSE: Proceedings of the Workshop on Software Engineering in Health Care; 2010; New York. New York: ACM; 2010. p. 89-99. http://dx.doi. org/10.1145/1809085.1809097

Corrêa C, Nunes FLS, Bezerra A, Carvalho Junior PM. Evaluation of VR medical training applications under the focus of professionals of the health area. In: ACM Symposium on Applied Computing: Proceedings of the 24th ACM Symposium on Applied Computing; 2009; Honolulu, Hawaii. New York: ACM; 2009. p. 821-5.

Costa RMEM, Mendonça I, Souza DS. Exploring the intelligent agents for controlling user navigation in 3D games for cognitive stimulation. In: ICDVRAT 2010: Proceedings of the 8th International Conference on Disability, Virtual Reality and Associated Technologies; 2010 Aug.; Viña del Mar, Chile. ICDVRAT Archive \& Online Proceedings; 2010. p. 1-6.

Costa RM, Carvalho L. The acceptance of virtual reality devices for cognitive rehabilitation: a report of positive results with schizophrenia. Computer Methods and Programs in Biomedicine. 2004; 73(3):173-82. http://dx.doi.org/10.1016/ S0169-2607(03)00066-X

Cunha A, Schenini F. Empresas têm pouca participação em pesquisas. Portal do Ministério da Cultura (MEC) [Internet]. 2007 [cited 201 set 16]. Available from: http://portal.mec.gov.br/index.php?option $=\mathrm{com}_{-}$ content $\&$ task $=$ view $\&$ id $=8580$

Cunha ÍLL, Monteiro BS, Moraes RM, Machado LS. AnatomI 3D: Um atlas digital baseado em realidade virtual para ensino de medicina. In: SBC Symposium on Virtual Reality: Proceedings of the SBC Symposium on Virtual Reality; 2006; Belém, Brasil. SBC; 2006. p. 3-14.

D’Angelo M, Narayanan S, Reynolds DB, Kotowski S, Page S. Application of virtual reality to the rehabilitation field to aid amputee rehabilitation: Findings from a systematic review. Disability and Rehabilitation: Assistive Technology. 2010; 5(2):136-42. http://dx.doi. org/10.3109/17483100903387622

DesRoches CM, Campbell EG, Rao SR, Donelan K, Ferris TG, Jha A, Kaushal R, Levy DE, Rosenbaum S, Shields AE, Blumenthal D. Electronic health records in ambulatory care - A national survey of physicians. New England Journal of Medicine. 2008; 359:50-60. PMid:18565855. http://dx.doi. org/10.1056/NEJMsa0802005

Dorsey ER, Roulet J, Thompson JP, Reminick JI, Thai A, White-Stellato Z, Beck CA, George BP, Moses III H. Funding of US Biomedical Research, 2003-2008. Journal of the Medical Association - JAMA. 2010; 302(2):137-43.

Garg KC. An overview of cross-national, national, and institutional assessment as reflected in the international journal. Scientometrics. 2003; 56(2):169-99. http://dx.doi. org/10.1023/A:1021963010621

Gerardi M, Rothbaum BO, Ressler K, Heekin M, Rizzo A. Virtual reality exposure therapy using a virtual Iraq: Case report. Journal of Traumatic Stress. 2008; 21(2):209-13. PMid:18404648. http://dx.doi.org/10.1002/jts.20331

Carvalho ACPLF, Brayner A, Loureiro A, Furtado AL, Von Staa A, Lucena CJP, Souza CS, Medeiros CMB, Lucchesi CL, Silva ES, Wagner FR, Simon I, Wainer J, Maldonado JC, Oliveira JPM, Ribeiro L, Velho L, Gonçalves MA, Baranauskas MCC, Mattoso M, Ziviani N, Navaux POA, Torres, RS, Almeida VAF, Meira Junior W, Kohayakaw Y. Grandes desafios da pesquisa em computação no Brasil - 2006-2016. Relatório Técnico [Internet]. 2006 [cited 2011 out 4]. Available from: http://www.ic.unicamp.br/ cmbm/ desafios_SBC/RelatorioFinal.pdf

Johnsen K, Raij A, Stevens A, Lind, DS, Lok B. The validity of a virtual human experience for interpersonal skills education. In: SIGCHI: Proceedings of the SIGCHI 
Conference on human factors in computing systems; 2007; New York. New York: ACM; 2007. p. 1049-58.

Kuljis J, Paul RJ, Stergioulas LK. Can health care benefit from modeling and simulation methods in the same way as business and manufacturing has? In: Conference on Winter Simulation: 40 years!: Proceedings of the 39th Conference on Winter Simulation; 2007; New Jersey. Piscataway: IEEE Press; 2007. p. 1449-53.

Lima L, Nunes FLS, Takashi R, Brega JRF, Sementille AC. Virtual reality for medical training: a prototype to simulate breast aspiration exam. In: ACM SIGGRAPH: Proceedings of the International Conference on Virtual-Reality Continuum and its Applications in Industry; 2004 Jun 16-18; Singapore. New York: ACM; 2004. p. 328-31.

Ma J, Nickerson JV. Hands-on, simulated, and remote laboratories: A comparative literature review. ACM Computing Surveys. 2006; 38(3):7. http://dx.doi. org/10.1145/1132960.1132961

Machado LS, Moraes RM, Nunes FLS, Costa RMEM. Serious games baseados em realidade virtual para educação médica. Revista Brasileira de Educação Médica. 2011; 35(2):254-62. http://dx.doi.org/10.1590/S0100-55022011000200015

Machado LS, Mello AN, Odone Filho V, Zuffo MK. Virtual reality simulation of pediatric bone marrow harvest for transplant. Medical and Pediatric Oncology. 2002; 39(4):282.

Machado LS, Moraes RM. Intelligent decision making in training based on virtual reality. In: Da Ruan, editor. Computational Intelligence in Complex Decision Systems. Paris: Atlantis Press; 2010. p. 85-123.

Machado LS, Moraes RM. VR-based simulation for the learning of gynaecological examination. Lecture Notes in Computer Science. 2006; 4282: 97-104. http://dx.doi. org/10.1007/11941354_11

Machado LS, Moraes RM, Souza DFL, Souza LC. A framework for development of virtual reality-based training simulators. Studies in Health Technology and Informatics. 2009; 142:174-6. PMid:19377142.

Moraes RM, Machado LS. Multiple assessment for multiple users in virtual reality training environments. Lecture Notes in Computer Science. 2007; 4756:950-6. http://dx.doi. org/10.1007/978-3-540-76725-1_98

Moraes RM, Machado LS. Fuzzy continuous evaluation in training systems based on virtual reality. In: IFSAEUSFLAT 2009: Proceedings of the International Fuzzy Systems Association World Congress; 2009; Lisbon, Portugal. Springer; 2009. p. 102-7.

Moraes RM, Machado LS. Development of a medical training system with integration of users' skills assessment. In: Jae-Jin Kim, editor. Virtual Reality. Viena: Intech; 2011. p. 325-48.

Nunes FLS, Costa RMEM. The virtual reality challenges in the health care area: a panoramic view. In: ACM Symposium on Applied Computing: Proceedings of 23th ACM Symposium on Applied Computing; 2008; New York. New York: ACM; 2008. p. 1312-16. v. 2.
Nunes FLS, Oliveira ACMTG, Rossato DJ, Machado MIC. ViMeTWizard: Uma ferramenta para instanciação de um framework de Realidade Virtual para treinamento médico. In: CLEI 07: Anais do XXXIII Conferencia Latinoamericana de Informática; 2007; San José, Costa Rica. Taller Latinoamericano de Informática para la Biodiversidad - INBI; 2007. p. 1-8.

Obana FY, Tori R. Conceitos de presença. In: WRVA 2010: Anais do VII Workshop de Realidade Virtual e Aumentada; 2010; São Paulo. Universidade Presbiteriana Mackenzie; 2010.

Oliveira ACMTG, Nunes FLS. Building a open source framework for virtual medical training. Journal of Digital Imaging. 2010; 23(6):706-20. PMid:19789924. PMCid:2978884. http://dx.doi.org/10.1007/s10278-0099243-3

Parsons T, Rizzo A. Affective outcomes of virtual reality exposure therapy for anxiety and specific phobias: A metaanalysis. Journal of Behavior Therapy and Experimental Psychiatry. 2008; 39(3):250-61. PMid:17720136. http://dx.doi.org/10.1016/j.jbtep.2007.07.007

Powell W, Stevens B, Hand S, Simmonds M. Sounding Better: Fast audio cues increase walk Speed in treadmillmediated virtual rehabilitation environments. Studies in Health Technology and Informatics. 2010; (154):202-7.

Rahman SA, Rahman A, Shaheen AA. Virtual reality use in motor rehabilitation of neurological disorders: A systematic review. Middle-East Journal of Scientific Research. 2011; 7(1):63-70.

Ramos FM, Nunes FLS. Construção de atlas de anatomia e fisiopatologia do câncer de mama utilizando realidade virtual. In: Simpósio Brasileiro de Computação Gráfica e Processamento de Imagens, Workshop de Teses de Dissertações em Computação Gráfica e Processamento de Imagens: Anais do XVIII Simpósio Brasileiro de Computação Gráfica e Processamento de Imagens, Anais do IV Workshop de Teses de Dissertações em Computação Gráfica e Processamento de Imagens; 2005; Natal, Brasil. SBC; 2005 .

Santos EP, Nunes FLS, Tori R, Nunes C. Definindo parâmetros para avaliação da aquisição de conhecimento em ambientes virtuais 3D. In: III WAValia: Anais do III Workshop sobre Avaliação e Acompanhamento da Aprendizagem em Ambientes Virtuais; 2010; João Pessoa, Brasil. SBA; 2010.

Tori, R, Kirner C, Siscoutto R. (2006) Fundamentos e tecnologia de realidade virtual e aumentada. Porto Alegre: SBC; 2006.

Tori R, Nunes FLS, Gomes VHP, Tokunaga DM. VIDA: Atlas anatômico 3D interativo para treinamento a distância. In: WCCE: Anais do XV Workshop Sobre Informática na Escola; 2009; Bento Gonçalves, Brasil. SBC; 2009.

Unesco. Unesco Science Report 2010 - The current status of science around the world. Unesco Publishing [Internet]. 2010 [cited 2011 Oct 4]. Available from: http://unesdoc.unesco. org/images/0018/001899/189958e.pdf 
Wainer J, Xavier EC, Bezerra F. Scientific production in Computer Science: A comparative study of Brazil and other countries. Scientometrics. 2009; 81(2): 535-47. http://dx.doi. org/10.1007/s11192-008-2156-y

Wang D, Zhang Y, Wu J. A Hierarchical model of motor skill for haptic-assisted virtual reality training. The International Journal of Virtual Reality. 2010; 9(3):75-81.
Wauke AP, Carvalho LAV, Costa RMEM. Tratamento de fobias urbanas por meio de ambientes virtuais. Arquivos Brasileiros de Psiquiatria, Neurologia e Medicina Legal. 2005; 99(4):5-11.

\section{Autores}

Fátima de Lourdes dos Santos Nunes

Laboratório de Aplicações de Informática em Saúde - LApIS, Escola de Artes, Ciências e Humanidades, Universidade de São Paulo - USP, Rua Arlindo Béttio, 1000, CEP 03828-000, São Paulo, SP, Brasil

\section{Rosa Maria Esteves Moreira da Costa}

Departamento de Informática e Ciência da Computação, Instituto de Matemática e Estatística, Universidade Estadual do Rio de Janeiro - UERJ, Rua São Francisco Xavier 524, 60 B1. B,

CEP 20550-013, Rio de Janeiro, RJ, Brasil

\section{Liliane dos Santos Machado}

Laboratório de Tecnologias para o Ensino Virtual e Estatística - LabTEVE, Departamento de Informática - CCEN, Universidade Federal da Paraíba - UFPB, Cidade Universitária s/n, CEP 58051-900, João Pessoa, PB, Brasil

\section{Ronei Marcos de Moraes}

Laboratório de Tecnologias para o Ensino Virtual e Estatística - LabTEVE, Departamento de Estatística - CCEN, Universidade Federal da Paraíba - UFPB, Cidade Universitária s/n, CEP 58051-900, João Pessoa, PB, Brasil 